\title{
Smooth Trajectory Generation with 4D Space Analysis for Dynamic Obstacle Avoidance
}

\author{
Suhyeon Gim ${ }^{1,2}$, Lounis Adouane ${ }^{1}$, Sukhan Lee $^{2}$ and Jean-Pierre Derutin ${ }^{1}$ \\ ${ }^{1}$ Institut Pascal/Université Blaise Pascal UMR6602, 63177, Aubiére Cedex, France \\ ${ }^{2}$ SungKyunKwan University, Seoburo 2066, 440-746, Suwon, Korea \\ \{Suhyeon.Gim, Lounis.Adouane, derutin\}@univ-bpclermont.fr, \{soohyunkim,lsh1\}@skku.edu
}

\begin{abstract}
Keywords: Continuous curvature path, dynamic obstacle avoidance, velocity planning.
Abstract: This paper presents smooth trajectory generation scheme for obstacle avoidance in static and dynamic environment. The smooth trajectory has successive two steps where smooth path is generated firstly and then corresponding velocity is planned along the path. Smooth path of continuous curvature is composed by parametrically adjusted clothoids with proposed algorithm and then the safe velocity planning is carried out in the 4D configuration framework. Two circles are used to completely surround the used nonholonomic car-like vehicle, this permit to check the probable future vehicle's collisions and to have space-time analysis. Some demonstrative simulations show the strong potential of the proposed smooth and flexible methodology for future experimentations with actual vehicles.
\end{abstract}

\section{INTRODUCTION}

Autonomous navigated vehicle or semi-autonomous driver assistant system has been attracting a lot of robotics research and industry fields for more than a decade. One of the most important issues is trajectory generation or path planning capability to manage a safe motion in a variety of risky environments. In path planning scheme, there are two kinds of planners (or planning algorithm) which are characterized either by global or local (Solea and Nunes, 2006), (Villagra et al., 2012). The global path planner gives a general navigational information (the path/trajectory to follow) to the vehicle to go from its initial configuration to one final configuration while taking into account all the obstacles in the environment (Khatib, 1986), (Giesbrecht, 2004). This planner use generally wholly known topological or grids-composed global map of the environment. The local path planner permits for its part to obtain only the navigational information from the current vehicle's configuration to a final configuration, which could be not the actual final destination of the vehicle, but just un intermediate configuration to avoid any obstructing obstacle. This last planner is considered more reactive (Kelly and Nagy, 2003), (Likhachev and Ferguson, 2009) in the sens that it can deal easily with dynamic obstacles (Chakravarthy and Ghose, 1998), (Fulgenzi et al., 2007). It is obviously important to notice that we could use a multitude of local planned path to obtain the global path for the navigation (Adouane, 2013).

The work presented in this paper focuses on local path planning algorithm with the consideration of dynamic obstacle avoidance. It is assumed that the vehicle has a predefined path from a global path planner and the local planner is required to generate a flexible and smooth trajectory in the presence of any obstructing dynamic obstacle. The path generated from the local path planner should be sufficiently accurate and smooth to be followed by the car-like vehicle.

To obtain accurate trajectory for a car-like vehicle, it is important for the planner to take into account: nonholonomic contraintes of the vehicle as well as its kinematic and dynamic contraints (Lamiraux and Laumond, 2001). There have been a lot of smooth path generation methods for vehicles. The works using continuous curvature path generations like those given in (Thompson and Kagami, 2005) are very efficient in the sense that they take into consideration the vehicle's parameters as well as passenger comfort.

There have been a lot of smooth path generation methods for the vehicle and the continuous curvature path has been focused for its close relationship with vehicle parameters and driving comfort (Montes et al., 2007), (Labakhua et al., ).

The continuous curvature path (CCP) has some advantages that the steering behavior of the vehicle is closely related to the curvature variation of the path 
to follow and the vehicle's movement is assured to be smooth along all the trajectory. This trajectory is especially useful for the nonholonomic car-like vehicle and the vehicle can follow the path without ever stopping to reorient its front wheels. The CCP for a nonholonomic vehicle are dealt with many previous works. Firstly, Dubins (Dubins, 1957), Reeds-Shepp (Reeds and Shepp, 1990) (RS path) proposed the smooth path model for nonholonomic vehicle yielding to the shortest in travel length, however it lacks the curvature continuity. Fraichard-Scheuer (Fraichard and Scheuer, 2004) (FS path) presented a continuous steering planning composed by lines, circular arcs and clothoids curves. This latter curves, with continuous curvature, take into account upper-bounded curvature and upper-bounded sharpness in the absence of obstacles. The work of (Wilde, 2009) presented a simple and fast trajectory generation method of continuous curvature with minimum sharpness for human natural and safe driving. However, the solution was limited only for the lane change maneuver example.

In this paper, a parametric Continuous Curvature Path (pCCP) which is proposed in (Gim et al., 2014 ) is extended to the problem of non-zero curvatures boundary conditions and it is applied to a simple dynamic obstacle avoidance problem by using the extended-pCCP or e-pCCP. The smooth trajectory generation for the static and dynamic obstacle avoidance is carried out by e-pCCP paths integrated with smooth velocity planning scheme. For e-pCCP, this work has different contributions with regard to the literatures. At first, compared to FS path (Fraichard and Scheuer, 2004), the proposed solution makes no limitation on the sharpness or curvature. FS path solves the zero curvatures configuration problem while fixing the sharpness in all clothoids or uses symmetric elementary paths by replacing the maximal curvatured arc segment of RS path with a maximal sharpness clothoid. Another point can be found that the nonzero curvature configurations on both ends are dealt with for obstacle avoidance or path replanning manouevre where initial and final curvature values are determined from current steering and obstacle boundary modelling. The nonzero initial curvature configuration is useful to replan a path while following pre-defined path with nonzero steering angle. The non-zero final curvature is also important to avoid the obstacle modeled in a bounded radius circle by generating the path for surrounding the obstacle circle. The other major point is introducing 4D configuration (Wu et al., 2011) to analyze the future vehicle's trajectory to determine the final avoidance pose and velocity set-points. Compared to the velocity obstacle model approach (Fiorini and Shiller,
1998), (Berg et al., 2008), (Wilkie et al., 2009), the proposed model analyzes all the expected collision cases for varying velocity of obstacles even for nonstraight trajectory. Resulting e-pCCP from 4D configuration analysis gives thus smooth trajectory even when dealing with dynamic obstacles. This paper is organized as follows. In the next section, the path following model for the nonholonomic vehicle and the problem is defined and then the proposed clothoids solutions for the trajectory generation are described with algorithmic description. In section 3, 4D configuration analysis is described with efficient vehicle's model and the smoothing procedure for the application to a practical implementation is addressed with simulated results. The paper ends with a conclusion with some prospects.

\section{PATH GENERATION FOR NONHOLONOMIC CAR-LIKE VEHICLE}

\subsection{Continuous curvature path model}

There is a useful mathematical representation for the nonholonomic car-like vehicle path according to its length (curvilinear abscissa) which it uses clothoid. A clothoid is represented by curvature variation with the length. Fully defined clothoidal form for a path is efficient for car-like vehicle in that it gives information on the curvature along the length and it could also give maneuvering information to the vehicle driver. A clothoid is defined by parametrical forms given by :

$$
\begin{gathered}
\kappa(s)=\kappa_{0}+\alpha s, \\
\theta(s)=\int_{0}^{s} \kappa(u) d u, \\
x(s)=\int_{0}^{s} \cos \theta(u) d u, \\
y(s)=\int_{0}^{s} \sin \theta(u) d u,
\end{gathered}
$$

where, $\alpha$ is the sharpness (or rate of curvature) for curvature $\kappa(s)$. Equation. (1) shows that the curvature increase or decrease by constant sharpness $\alpha$ and the orientation in (2) changes with integration of curvature in (1). It is to be noted that this mathematical relation is the same as for the physical relation between the steering angle and the vehicle orientation where the orientation varies in integrated amount of steering changes. It is also noted that the position in coordinate is determined only after the orientation at lengths 
is calculated as in (3), (4). This means that when one tries to find a sharpness for a clothoid to meet the desired position and orientation at a certain curvilinear distance requires to have an analytic model of the inverse kinematic solution, which is not yet available in the literature, because the problem is too complex to resolve.

A clothoid has the property to have continuous curvature which is either increasing or decreasing through the length. There are some kinds of formulations for the rate of the curvature such as polynomials, exponential or trigonemetric function, however, the $1^{\text {st }}$ order form (like what is given in equation (1)), relying on the constant sharpness, is well-known not only for its computational simplicity but also for phenomenal similarity to the real vehicle actuation system. To be more specific, the curvature of a point on the path corresponds to the steering angle of the vehicle which follows the path at the point and the sharpness signifies the rate of the steering change at that point. In the next subsection, the Problem definition and algorithmic solution will be given, while beginning by recalling the already proposed algorithm in (Gim et al., 2014).

\subsection{Parametric clothoid for path generation}

This subsection deals with a problem to generate a smooth path in local planner for a nonholonomic car-like vehicle. The local planner generates a short path in the detectable range distance and its boundary condition is defined by two configurations, i.e. initial $P_{i}$ and final $P_{f}$. For CCP generation, clothoid is the major segment along the path and each clothoid is linked to other clothoids while satisfying boundary configurations with attributed constraints in vehicle. Before to explain the main contribution of the proposed paper, let us remind what was already proposed in (Gim et al., 2014), which consist on an algorithmic approach to compute the parameters of the clothoid which has as initial configuration $P_{i}$ and as a final configuration $P_{f}$. It is to be noted that the initial and final specified set-poits curvatures are equal to zero. The work considered thus the initial and the final steering angle of the front wheels $(\kappa$ in (1)) are always zero, which means that the vehicle start from straight line and finish also with straight line. The proposed algorithm in (Gim et al., 2014), called parametricContinuousCurvaturePath (pCCP) permits to compute these parameters based on some basic properties or pattern of Clothoids. Indeed, when a clothoid is defined, the relations among its parameters are given as following.

$$
\kappa=\sqrt{2 \delta \alpha}, \delta=\frac{\kappa^{2}}{2 \alpha}, s=\sqrt{\frac{2 \delta}{\alpha}},
$$

where all parameters are the values at the end point of the clothoid and $\delta$ means the amount of orientation change through the whole length which is called deflection (Labakhua et al., ). Note that $\alpha, \kappa$, $\delta$ and $s$ are closely related to each other and furthermore, if two of them are defined, then the others are also determined. This relation is important to make a clothoid meet a desired pose by adjusting independent two parameters, e.g. $(\alpha, \kappa)$ or $(\alpha, \delta)$. To specify the convergence criteria as well as determining initial values for the algorithm, some basic properties or pattern according to the parameter variation are useful to understand. To summarize the links between the parameters, 3 properties can be concluded:

Property 1 As the sharpness $\alpha$ increases with other parameters constant, the clothoid shrinks.

Property 2 As the deflection $\delta$ increases with other parameters constant, the clothoid expands.

Property 3 As the curvature $\kappa$ increases with other parameters constant, the clothoid winds up inward.

The path in the problem requires at least two clothoids to satisfy the both configurations where each clothoid has a unique sharpness to adjust its curvature to maximal value, i.e. $C_{s}: s \mapsto\left[s_{0}, s_{\ell}\right]$. When a clothoid is defined as $C_{1}$, the other as $C_{2}$ and they are composed as $C_{1} \oplus C_{2}$, while satisfying the orientation continuity $G^{1}$ as well as curvature continuity $G^{2}$ at the connection point where $G^{n}$ is $n$-th order of Geometric Continuity.

\subsection{Problem definition with algorithmic solution}

In this paper, we make a focus on the smooth local path generation for obstacle avoidance where the initial and final curvatures are non-zero. This problem was not addressed in the former work (Gim et al., 2014) and the parametric convergence criteria in the algorithm also needs to be differently treated. Another importance for the problem comes from the question on how to cope with dynamic obstacle. When a vehicle follows the generated path for a static obstcle, the path should be replanned with current steering angle of initial non-zero curvature as well as nonzero steering angle at the final avoidance pose. To cope with the dynamic obstacle, it needs to be extended for pCCP. The curvature for initial configuration is given according to the current steering angle of the vehicle and the curvature for final configuration is determined from the obstacle boundary 
radius to generate the path that surrounds the moving obstacle boundary to avoid it.

To formalize the addressed problem, the following formulation is given.

Problem: From $P_{i}\left(x_{i}, y_{i}, \theta_{i}, \kappa_{i}\right)$ to $P_{f}\left(x_{f}, y_{f}, \theta_{f}, \kappa_{f}\right)$ with $\kappa_{i} \geq 0, \kappa_{f} \neq 0$, find the minimum number of clothoids which satisfy the both configurations with curvature continuity along the path.

Proposition 1. The configuration is defined on the first quarter plane of the Cartesian coordinates and other configurations can be defined by symmetric manner to its $X-Y$ coordinates. The initial pose is on the origin with its orientation $\theta_{i}=\pi / 2$ and the final pose exists on the first quarter of Cartesian coordinate.i.e. $x>0, y>0$.

Proposition 2. The curvature in a clothoid has positive sign when the steering angle is in the right hand side from its middle origin, and vice versa. The sharpness in a clothoid has positive sign when the steering turns right (clockwise) noted as $C^{R}$ and vice versa as $C^{L}$.

With the above propositions, the pose configurations of the problem can be slightly changed by $P_{i}\left(0,0, \frac{\pi}{2}, \kappa_{i} \geq 0\right)$ and $P_{f}\left(x_{f}, y_{f}, \theta_{f}, \kappa_{i} \neq 0\right)$.

Note that there are two cases according to the turning direction or curvature sign at the final pose.

$$
\left\{\begin{array}{l}
\kappa_{f}>0: C_{i}^{R} C_{f}^{R} \\
\kappa_{f}<0: C_{i}^{R} C_{f}^{L}
\end{array}\right.
$$

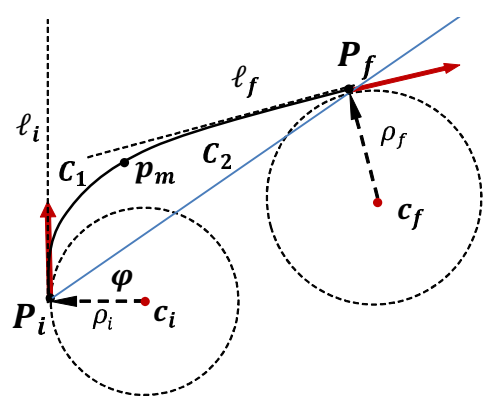

(a) Two clothoids composition

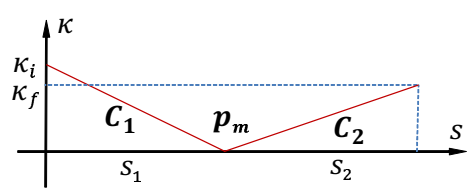

(b) Curvature diagram

Figure 1: e-pCCP problem : $C_{1}^{R} C_{2}^{R}$

Figure 1 and Figure 2 show the path comprised by two clothoids composition with initially same curvature but with different turning direction as noted by

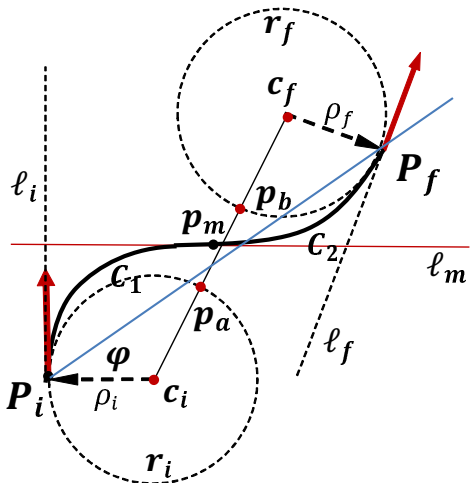

(a) Two clothoids composition

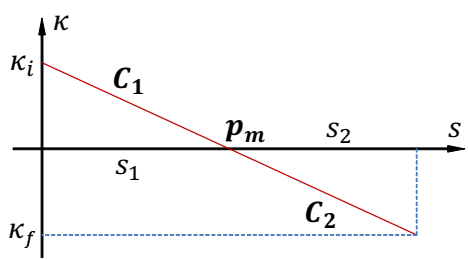

(b) Curvature diagram

Figure 2: e-pCCP problem : $C_{1}^{R} C_{2}^{L}$

$\kappa_{f}>0$ (cf. Figure 1) and $\kappa_{f}<0$ (cf. Figure 2) with its corresponding curvature diagram respectively.

The initial pose $P_{i}$ and the final pose $P_{f}$ are denoted by vector from each end position with given curvatures (or radius of curvature) $\rho_{i}$ and $\rho_{f}$ where the extension lines are displayed from each pose as $\ell_{i}$ and $\ell_{f}$ respectively (cf. Figure 2(a)). While fulfilling the pose configuration at both ends, clothoid segment $C_{1}$ and $C_{2}\left(\forall s \in\left[s_{0}, s_{l}\right]\right)$ are generated and located for its final end $C_{1}\left(s_{l}\right)$ to be $P_{i}, P_{f}$ with same orientatation of $\ell_{i}$ and $\ell_{f}$. The connection point $p_{m}$ where each orientation and curvature are same for geometric continuity is found from a parametrical adjustment and the point can be a line depending on the configuration. Corresponding curvature diagram of $C_{1}^{R} C_{2}^{R}$ is shown in Figure 1(b) with convention described in Proposition 2. This diagram features the steering behavior while following the path, in other words, the steering turns left from the right side position to its center and then turns right to make another right position in steering angle. Two clothoids designate two turning motions for steering angle and it certifies the motion is continuous through all the travel length as shown in curvature diagram.

Another case $C_{1}^{R} C_{2}^{L}$ is shown in Figure 2. The clothoid generation and composition procedure are the same then the case of $C_{1}^{R} C_{2}^{R}$ while satisfying the configuration with curvature continuity. However, different to first case, the solution of Figure 2(a) is 
only available where there is sufficient space for the line $\ell_{m}$ to pass by between two points $p_{a}, p_{b}$ that the line $\overline{c_{i} c_{f}}$ and circles $r_{i}, r_{f}$ meet each other. $\left(c_{i}\right.$, $c_{f}$ are the center position of circle $r_{i}, r_{f}$.) such that $\left|c_{i}-c_{f}\right|>\rho_{i}+\rho_{f}$ as a necessary condition.

In this case, it is required that a clothoid is supplemented to satisfy the two clothoids configuration.

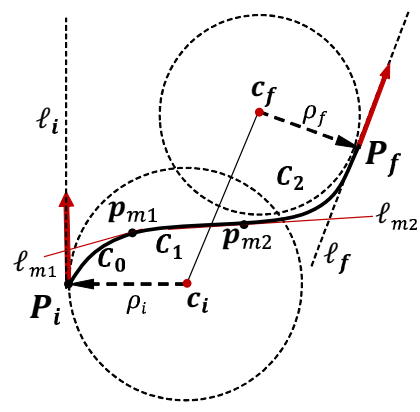

(a) Three clothoids composition

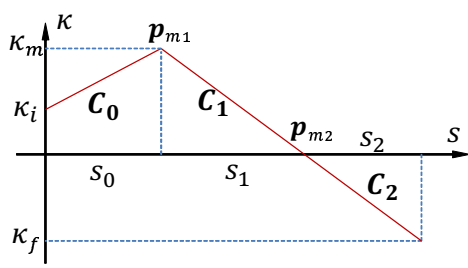

(b) Curvature diagram

Figure 3: e-pCCP problem : $C_{0}^{R} C_{1}^{R} C_{2}^{L}$

Figure 3 depicts the additional case $C_{0}^{R} C_{1}^{R} C_{2}^{L}$ including $C_{0}$ where both radius of end curvatures are overlapped so that two clothoids can not make a meet with each other. One of feasible method to tackle the problem is to add a clothoid which increase from the given initial curvature $\kappa_{i}$ to any higher curvature $\kappa_{m}$ as shown in Figure 3(a). The following curvature diagram of (b) explains the curvature variation of three clothoids. The resulting path of three clothoids is useful for the dynamic obstacle planning when the current curvature is so low (including the case $\kappa_{i} \simeq 0$ ) that it is expected any collision with the obstacle which is dealt with the demonstrative examples in section 4.

The detailed procedure for generating clothoids is described in Algorithm 1 for two clothoids $C_{1}$ and $C_{2}$ that covers the cases $C_{i}^{R} C_{f}^{R}$ and $C_{i}^{R} C_{f}^{L}$ of (6).

In Algorithm 1, both end curvatures $\kappa_{i}, \kappa_{f}$ are given and initial deflection $\delta_{i}$ for $C_{1}$ with variations $d \delta, d \rho$ are assumed by designer before entering the loop. In line 3 , the parameter is varied according to the property found as Properties 1 to 3 and each clothoid is generated as given in the line 4 , line 6 by (5). The variation determinant $\lambda\left(C_{2}\left(s_{0}\right)\right)$ for $d \delta$ is important for fast convergence with small number of it- erations. Note that the point $C_{2}\left(s_{0}\right)$ is at the other end of $C_{2}$ since the curvature value in a clothoid is varied from 0 to its maximum $\kappa_{\max }$ by $s_{0}$ to $s_{\ell}$ variation. The rule for determining the sign of $d \delta$ is defined by an inequality condition which checks on the point $p(x, y)$ whether it locates in upper or lower side of the tangential line at $p_{0}\left(x_{0}, y_{0}\right)$ as following.

$$
\lambda\left(p, p_{0}\right)=-\tan \left(\theta_{i}-\delta_{1}\right) x+y+x_{0}+\tan \left(\theta_{i}-\delta_{1}\right) y_{0}
$$

where it is a straight line passing the end point of a clothoid $C_{1}$, i.e. $C_{1}\left(s_{0}\right)$ and $\lambda\left(C_{2}\left(s_{0}\right)\right)$ is checked for the location on the point $C_{2}\left(s_{0}\right)=(x, y) \mid C_{2}, s=s_{0}$. The other determinant equation is $\lambda^{\perp}$ which is the perpendicular line of $\lambda\left(C_{2}\left(s_{0}\right)\right)$ which is described as,

$$
\lambda^{\perp}\left(p, p_{0}\right)=x-x_{0}-\tan \left(\theta_{i}-\delta_{1}\right) y_{0}+\tan \left(\theta_{i}-\delta_{1}\right) y .
$$

The composition of two clothoids by given constraints requires only one adjusting variable for solving the problem and there are unsolvable configurations only by regulating the variable such as the end point of $C_{1}$ locates inside of the final radius of curvature circle $r_{f}$. In such case, additional regulation is performed to make two clothoids meet by reducing the radius $\rho_{f}$ (or increasing the curvature) at the final pose as described in line 13. The convergence toward the solution is defined in line 4 where $D^{e}$, the distance between the composed curve $C_{1,2}$ and $\ell_{m}$ reach the threshold $\varepsilon$, e.g. $10^{-3}[m]$.

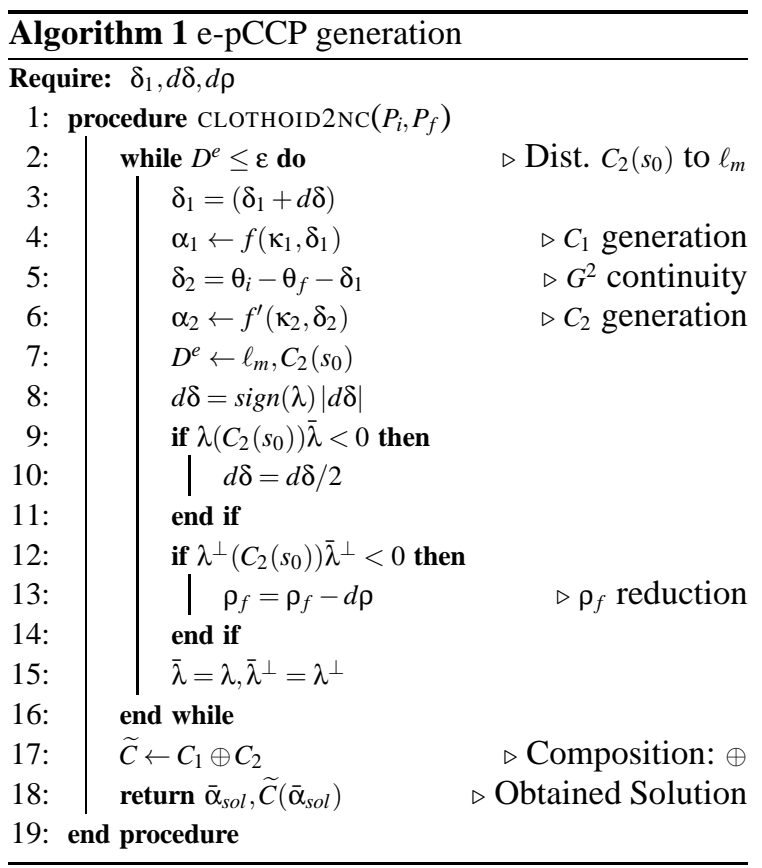




\section{VELOCITY PLANNING IN 4D SPACE}

\subsection{D space analysis for obstacle avoidance}

In this subsection, a framework of 4D space is introduced to analyze collision detection in space and time domain and to plan the obstacle avoidance maneuver for a vehicle. Cartesian coordinated 3D configuration space (C-space) with additional time axis are constructed to analyze future status for dynamic objects. A representative description is shown in Figure 4 where two vehicle move in each direction and expected to make collision on intersection at a collision time $t_{c}$ by meeting the two future trajectory lines $L_{a}$ and $L_{b}$. It is shown that an obstacle vehicle moving in velocity $\mathrm{v}_{a}$ and a vehicle of $\mathrm{v}_{b}$ collide at the state $S_{c}$ in 4D space. In 4D space, it is easy to check on any possible collision among dynamic objects by measuring minimum distance between each future trajectory line whether the resultant value is less than a collision threshold distance where the threshold is bounded by object modeling scheme.

With 4D analysis and circular representation for the vehicle, feasible avoidance pose can be determined to avoid any collision by replanning the trajectory. One of possible avoidance plan is to modify the velocity profile by encompassing the collision boundary with minimal changes of original velocity profile. The other possible avoidance consists to set up the arrival pose of the future generating path to be safe from any collision. For the dynamic obstacle avoidance, here, two avoidance poses (front and rear) are considered as follows.

Figure 5 presents the collision plane of the obstacle where the horizontal axis corresponds to the configuration space $\mathbb{C}$ and vertical axis presents fu-

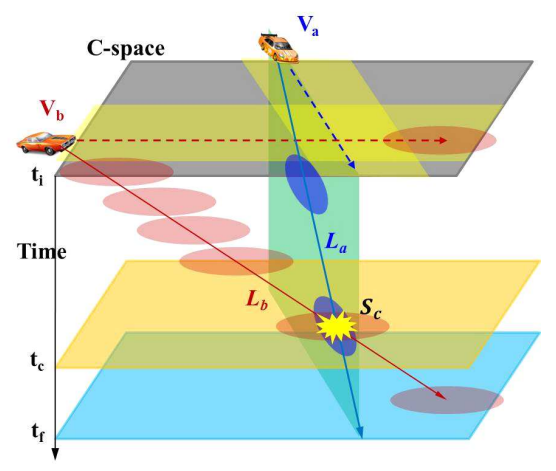

Figure 4: 4D configuration analysis for collision avoidance

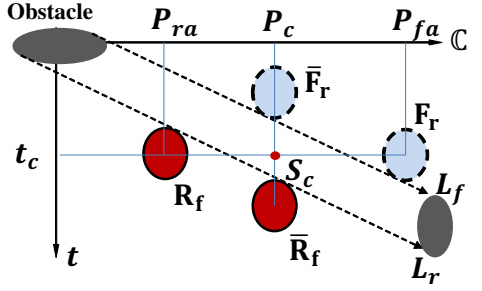

Figure 5: Avoidance pose planning in collision plane

ture time. Consistent with the problem definition, the obstacle is assumed to move from left side of the line $\ell_{i}$ (cf. Figures 1,2 and 3) to right side with constant velocity (same speed and orientation) at $t_{1}$. This methodology remains effective when the obstacle changes its orientation or speed such that replanning is started as the change is detected. At the position of $P_{c}$ in $t_{c}$, a collision is expected at the state $S_{c}$. In the plane, there are two avoidance modes according to the location of the obstacle boundaries. Upper region of the boundary, noted as $L_{f}$ corresponds to front avoidance region and lower part of the boundary $L_{r}$ corresponds to rear avoidance. The avoidance pose (or final pose) $P_{f}$ is determined to be the point on the tangential line which is perpendicular to obstacle velocity direction. According to the avoidance pose, the path by the vehicle is $C_{1}^{R} C_{2}^{R}$ for $L_{r}$ and $C_{1}^{R} C_{2}^{L}$ for $L_{f}$ avoidance. The vehicle is represented by two circles in this work to simply check the collision distance with obstacles. When the obstacle comes close to collide with the vehicle from the right side to left of $\ell_{i}$ (cf. Figures 1,2 and 3), the avoidance modes are changed each other with the same scheme. Under two avoidance shemes of velocity replanning and path replanning, there are four avoidance statuses $\bar{F}_{r}, \bar{R}_{f}, F_{r}$ and $R_{f}$ where every subscript signifies colliding obstacles (circle) with one of the two circles (which surround the vehicle).

Note that $\bar{F}_{r}, \bar{R}_{f}$ in velocity change avoidance locate at the vertical line of $P_{c}$ where both circles pass by the expected collision position $P_{c}$ but with arrival time differences. For the path replanning, it requires another stragegy to constrain the arrival time at rear avoidance arrival pose $P_{r a}$ or front avoidance arrival pose $P_{f a}$. In what follows, the strategy to locate the collision time $t_{c}$ to be out of the obstacle boundary is to find the time at avoidance pose $F_{r}$ or $R_{f}$ which are in the same level of $t_{c}$ as well as being closest to the obstacle boundary. In the avoidance maneuver, the initial velocity at $t_{i}$ could be maintained with a little changes according to the changes in the travel length of the new replanned path. 


\subsection{Dynamic obstacle avoidance}

With the obtained solution for path planning, using clothoids given in section 2.3, it is proposed in this section to apply it for dynamic obstacle avoidance problem. In the dynamic obstacle problem, the obstacle is represented with a circle (Chakravarthy and Ghose, 2011) having constant velocity.

With the configuration described in section 2.3, epCCP to avoid obstacles is generated.

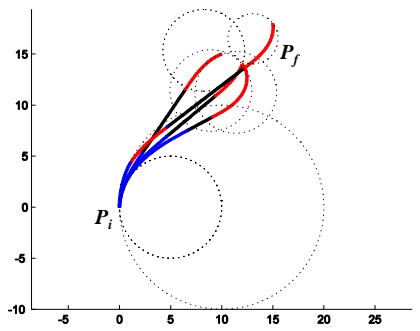

Figure 6: e-pCCP for obstacle avoidance

In Figure 6, four different configurations are applied for e-pCCP generation. Each generated path surround the obstacle circle to avoid the obstacle by path following. The initial configuration and final configuration are denoted by $P_{i}$ and $P_{f}$ while the corresponding radius of curvatures are presented respectively. The generated paths are composed by two clothoid with additional straight line to have curvature continuity while avoiding the obstacle boundary as close as possible. This avoidance path has additional advantages as follows. At first, the path could be short accoring to the static or dynamic obstacle. Secondly, the rate of steering turn (or sharpness) is minimal compared to farther pose avoidance from the obstacle. Lastly, such path could be still efficient when the obstacle changes its moving direction while avoidng since the path could be also regenerated with current steering angle as initial curvature and new final configuration by expected obstacle motion. Obtained epCCP is applied in 4D configuration space in order that the path combines the velocity smoothness for dynamic obstacle to fulfill the continuous trajectory of vehicles.

Figure 7 presents a demonstrative 4D space simulation for dynamic obstacle avoidance. In (a), the obstacle trajectory of A plane and the vehicle trajectory of $\mathbf{B}$ plane are plotted in the space by given information for the obstacle $P_{i}^{A}(-6,10,0,0)$ with $0.6 \mathrm{~m} / \mathrm{s}$ and the vehicle $P_{i}^{B}\left(0,0, \frac{\pi}{2}, 0.08\right)$. In this configuration, a collision is predicted at $10 \mathrm{sec}$ in future and (b) depicts the obstacle avoidance poses determination as described in Figure 5.

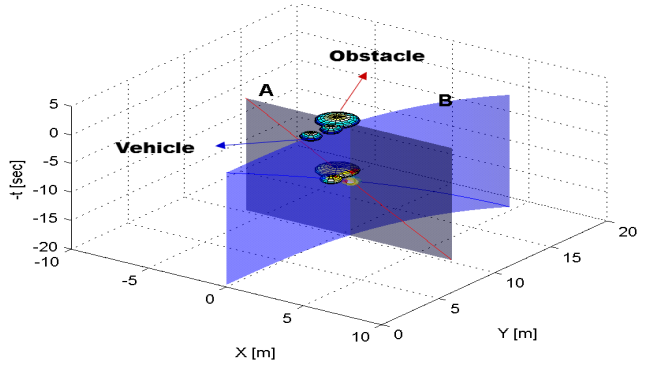

(a) Collision case

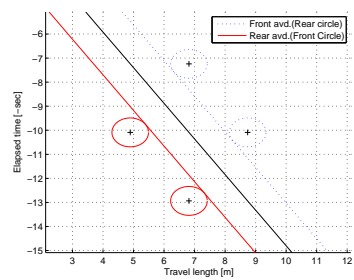

(b) Avoidance planning

Figure 7: 4D analysis for dynamic obstacle avoidance

Figure 8 shows the replanned trajectory for the simulation given in Figure 7 where (a) is the velocity replanning and (b) is the replanned path by e-pCCP. In (a), collision state $S_{c}$ is shown along the trajectory line $v_{c}$ with obstacle trajectory $v_{o b}$. For the ve-

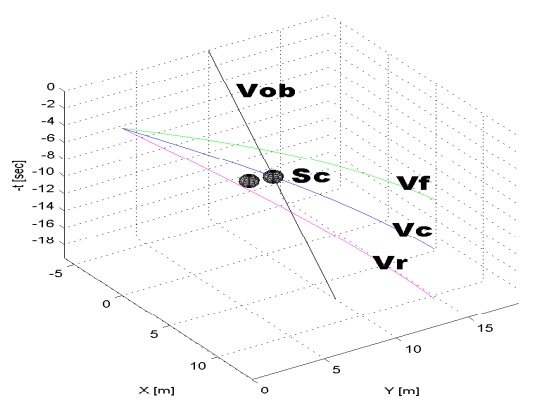

(a) Velocity replanning

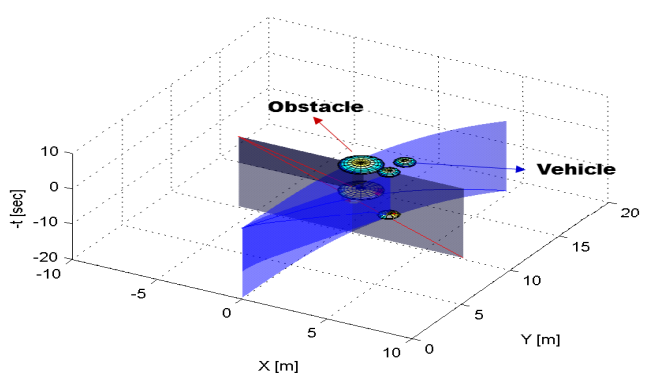

(b) Safe trajectory

Figure 8: e-pCCP for obstacle avoidance 
hicle, the front avoidance with current velocity $v_{f}$ and rear avoidance with current velocity $v_{r}$ are planned while both avoidance trajectories keep the safe distance to avoid any collosion with the obstacle trajectory. Figure 8(b) demonstrates that the generated trajectory guarantees a smooth collision avoidance with the dynamic obstacle.

\section{CONCLUSIONS}

This paper presents a smooth trajectory generation for dynamic obstacle with 4D space analysis and e-pCCP. Extending $\mathbf{p C C P}$ to the nonzero curvatures problem permits the generated path to be reactive for dynamic obstacle. By collision checking in 4D space analysis with two circles vehicle representation for the vehicle, the avoidance poses are determined to avoid any risk of collision on the future. The resultant trajectory has steering smoothness on the path as well as smooth velocity changes along the path. Demonstrative examples on a dynamic obstacle shows the effectiveness of the proposed methods and expected to be implemented for more complicated dynamic environments such as multiple obstacles or cluttered areas with real time performance.

\section{REFERENCES}

Adouane, L. (2013). Toward smooth and stable reactive mobile robot navigation using on-line control set-points. In IEEE/RSJ, IROS'13, 5th Workshop on Planning, Perception and Navigation for Intelligent Vehicles, Tokyo-Japan.

Berg, J. V., Lin, M., and Manocha, D. (2008). Reciprocal velocity obstacles for real-time multi-agent navigation. IEEE Int. Conf. on Robotics and Automation, pages 1928-1935.

Chakravarthy, A. and Ghose, D. (1998). Obstacle avoidance in a dynamic environment: A collision cone approach. IEEE trans. on Systems, Man and Cybernetics-Part A: Systems and Humans, 28(5):562-574.

Chakravarthy, A. and Ghose, D. (2011). Collision cones for quadric surfaces. IEEE Trans. on Robotics, 27(6):1159-1166.

Dubins, L. E. (1957). On curves of minimal length with a constraint on average curvature, and with prescribed initial and terminal positions and tangents. American Journal of Mathematics, 79:497-516.

Fiorini, P. and Shiller, Z. (1998). Motion planning in dynamic environments using velocity obstacles. Int. J. of Robotics Research, 17(7):760-772.

Fraichard, T. and Scheuer, A. (2004). From Reeds and Shepp's to continuous curvature paths. IEEE Trans. on Robotics, 20:1025-1035.
Fulgenzi, C., Spalanzani, A., and Laugier, C. (2007). Dynamic obstacle avoidance in uncertain environment combining pvos and occupancy grid. IEEE Int. Conf. on Robotics and Automation, pages 1610-1616.

Giesbrecht, J. (2004). Global path planning for unmanned ground vehicles.

Gim, S., Adouane, L., Lee, S., and Derutin, J.-P. (2014). Parametric continuous curvature trajectory for smooth steering of the car-like vehicle. Int. Conf. on Intelligent Autonomous Systems.

Kelly, A. and Nagy, B. (2003). Reactive nonholonomic trajectory generation via parametric optimal control. The International Journal of Robotics Research, 22(7 - 8):583-601.

Khatib, O. (1986). Real-time obstacle avoidance for manipulators and mobile robots. The int. J. of Robotics Research, 5(1):90-98.

Labakhua, L., Nunes, U., Rodrigues, R., and Leite, F. S. Smooth trajectory planning for fully automated passengers vehicles: Spline and Clothoid based methods and its simulation.

Lamiraux, F. and Laumond, J. P. (2001). Smooth motion planning for car-like vehicles. IEEE Trans. on Robotics and Automation, 17(4):498-501.

Likhachev, M. and Ferguson, D. (2009). Planning long dynamically feasible maneuvers for autonomous vehicles. Int. Journal of Robotics Research, 28(8):933945.

Montes, N., Mora, M. C., and Tornero, J. (2007). Trajectory generation based on rational Bezier curves as clothoids. IEEE Intel. Vehicles Symposium, pages 505-510.

Reeds, J. A. and Shepp, L. A. (1990). Optimal paths for a car that goes both forwards and backwards. Pacific Journal of Mathematics, 145(2):367-393.

Solea, R. and Nunes, U. (2006). Trajectory planning with velocity planner for fully-automated passenger vehicle. Intel. Transportation Systems Conf., pages 474480

Thompson, S. and Kagami, S. (2005). Continuous curvature trajectory generation with obstacle avoidance for car like robot. Int. Conf. on Computational Intelligence for Modeling, Control and Automation, pages 863-870.

Villagra, J., Milantes, V., Perez, J., and Godoy, J. (2012). Smooth path and speed planning for an automated public transport vehicle. Robotics and Autonomous Systems, 60:252-265.

Wilde, D. K. (2009). Computing clothoid segments for trajectory generation. IEEE/RSJ Int. Conf. on Intel. Robots and Systems, pages 2440-2445.

Wilkie, D., van den Berg, J., and Manocha, D. (2009). Generalized velocity obstacles. IEEE Int. Conf. on Intelligent Robots and Systems, pages 5573-5578.

Wu, P. P. Y., Campbell, D., and Merz, T. (2011). Multiobjective four-dimenstional vehicle motion planning in large dynamic environments. IEEE Trans. on Systems, Man, and Cybernetics-Part B:Cyberntetics, 41(3):621-634. 